\title{
Peritumoral administration of GPI-anchored TIMP-1 inhibits colon carcinoma growth in Rag-2 $\gamma$ chain-deficient mice
}

\author{
Matthias C. Raggi1,a, Roghieh Djafarzadeh2,a, \\ Niklas Muenchmeier², Monika Hofstetter', \\ Birgit Jahn², Nicole Rieth ${ }^{2}$ and Peter J. \\ Nelson ${ }^{2, *}$ \\ ${ }^{1}$ Surgery Clinic, Technical University Munich, \\ Ismaninger Str. 22, D-81675 Munich, Germany \\ ${ }^{2}$ Ludwig Maximilians University Munich, Medical \\ Policlinic, Schillerstr. 42, D-80336 Munich, Germany \\ ${ }^{\star}$ Corresponding author \\ e-mail: peter.nelson@med.uni-muenchen.de
}

\begin{abstract}
Exogenous application of recombinant TIMP-1 protein modified by addition of a glycosylphosphatidylinositol (GPI) anchor allows efficient insertion of the fusion protein into cell membranes. This 'cell surface engineering' leads to changes in the proteolytic environment. TIMP1-GPI shows enhanced as well as novel in vitro biological activities including suppression of proliferation, reduced migration, and inhibition of invasion of the colon carcinoma cell line SW480. Treatment of SW480 tumors implanted in Rag (-/-) common $\gamma$ chain (-/-) C57BL/6 mice with peritumorally applied TIMP-1-GPI, control rhTIMP-1 protein, or vehicle shows that TIMP-1-GPI leads to a significant reduction in tumor growth.
\end{abstract}

Keywords: colorectal cancer; matrix metalloproteinases; proliferation; TIMP-1-GPI; tissue inhibitors of metalloproteinases.

The matrix metalloproteinases (MMPs) are a family of zinc-containing endopeptidases that degrade components of the extracellular matrix (ECM) and also regulate various cellular functions through their post-translational modification (cleavage) of proteins (Nagase et al., 2006). The MMPs are represented by collagenases, gelatinases, stromelysins, and membrane-type MMPs (Visse and Nagase, 2003; Nagase et al., 2006). These enzymes have also been implicated in multiple stages of cancer progression, including the acquisition of an invasive phenotype and tumor proliferation. In colon carcinoma, expression of MMP-2, MMP-9, and MMP-7 have been associated with the general aggressiveness of the tumor (Matsuyama et al., 2002; Rath et al., 2006; Oshima et al., 2008). The increased expression of MMP-2 and MMP-9 has been linked to enhanced invasion, migration, and

a These authors contributed equally to this work. attachment of the colon carcinoma cell line SW480 (Murray et al., 2004). In contrast to other MMP family members, MMP-7 is usually overproduced by carcinoma cells as opposed to stromal cells and thus an involvement of this proteinase in tumorigenesis including that of colorectal cancer has been proposed (Yoshimoto et al., 1993; Cockett et al., 1994; Nagashima et al., 1997).

MMP activities are regulated at multiple levels, including endogenous inhibitors - the tissue inhibitors of metalloproteinases (TIMP-1, TIMP-2, TIMP-3, and TIMP-4) (Maskos and Bode, 2003). The balance between MMP and TIMP expression helps dictate ECM turnover and remodeling in normal tissue homeostasis and pathogenesis, and also moderates the regulatory functions of MMPs (Visse and Nagase, 2003; Nagase et al., 2006). The TIMP proteins have also been shown to have additional biological functions not directly attributed to MMP inhibition (Chirco et al., 2006; Nagase et al., 2006). These include the regulation of apoptosis, cell proliferation, and aspects of angiogenesis. We have previously demonstrated that the bioactivities of TIMPs can be altered by changing their cellular distribution (Djafarzadeh et al., 2004, 2006, 2009). The modification of TIMP-1 protein by the addition of a glycosylphosphatidylinositol (GPI) anchor allows the protein to be efficiently transferred from one cell surface to another (Medof et al., 1996). Exogenous application of recombinant TIMP-1-GPI fusion protein leads to efficient incorporation of TIMP-1 into cell surface membranes. This surface engineering with TIMP-1GPI has been shown to lead to enhanced as well as novel biological activities (Djafarzadeh et al., 2004, 2006, 2009).

We have previously identified a series of potential antitumor effects moderated by treatment with TIMP-1-GPI in an in vitro setting, including the sequestering of MMPs on the cell surface, reduced tumor secretion of MMPs, a dose-dependent inhibition of renal cell carcinoma proliferation, migration, invasion, and an increase in the sensitivity of the tumor cells to FAS-mediated apoptosis (Djafarzadeh et al., 2006, 2009). In the present study, we sought to verify a select set of these effects in vitro in a colon carcinoma cell line, and to then determine if these beneficial effects on tumor growth could also be reiterated in an in vivo setting. We specifically sought to evaluate the potential influence of peritumoral administration of the agent on tumor growth. This was performed in the absence of an adaptive immune response [Rag2 $\gamma$ chain (-/-) mice] (Shinkai and Alt, 1994) to exclude potential T cell suppression of tumor growth (Djafarzadeh et al., 2006).

The colon adenocarcinoma, grade III-IV tumor cell line SW480, was used as the model system (Murray et al., 
2004). The effects of treatment of the cells were first verified in vitro. The incorporation of recombinant TIMP-1GPI protein into the cell membrane of SW480 cells was tested by incubation with $14 \mathrm{ng} / \mathrm{ml}$ of purified TIMP-1GPI, recombinant human rhTIMP-1 control protein or vehicle for $1 \mathrm{~h}$ at $37^{\circ} \mathrm{C}$. Surface-associated TIMP-1 protein was detected using human specific TIMP-1 monoclonal antibody and fluorescence-activated cell sorting (FACS) (Djafarzadeh et al., 2006). Addition of vehicle or control rhTIMP-1 did not lead to a change in the FACS shift; however, GPI-anchored TIMP-1 resulted in a strong surface signal for TIMP-1 (Figure 1A).

The dose-dependent effect of an increasing surface level of TIMP-1 on the constitutive release of MMPs from the SW480 cells was evaluated (Figure 1B and C). Secretion of MMP-9 and MMP-2 protein from the SW480 cells was tested by gelatinase zymography (Klier et al., 2001; Djafarzadeh et al., 2004). The cells produced low but detectable levels of MMP-9 but undetectable levels of MMP-2. rhTIMP-1 protein at $14 \mathrm{ng} / \mathrm{ml}$ had no effect on MMP-9 secretion. In contrast, starting at $0.875 \mathrm{ng} / \mathrm{ml}$, TIMP-1-GPI treatment led to a concentration-dependent decrease of MMP-9 release into the growth media (Figure 1B). In the experiments presented, the growth media was pretreated with $1 \mu \mathrm{M}$ p-aminophenylmercuric acetate (APMA) to induce activation of proMMP-9 to MMP-9 for better quantification. Parallel zymography experiments demonstrated that the secreted enzyme was primarily the pro-form of the enzyme (data not shown).

MMP-7 plays multiple roles in the invasion and metastasis of carcinoma cells (Strand et al., 2004; Mylona et al., 2005). The cleavage of cell surface proteins other than ECM components by this metalloproteinase may also contribute to its tumorigenic effects. For example, MMP-7 has been shown to promote the activation of the epidermal growth factor (EGF) receptor by inducing the release of an EGF ligand (Mimori et al., 2004).

The secretion of MMP-7 from SW480 was tested by Western blot analysis of the growth media using monoclonal antibodies directed against human MMP-7. Culture media (serum-free) were taken $24 \mathrm{~h}$ after either no treatment of SW480, treatment with vehicle, $14 \mathrm{ng} / \mathrm{ml}$ of either rhTIMP-1 or TIMP-1-GPI or equal molar concentrations of GPI-anchor/phosphatidylserine, and then tested for the presence of MMP-7. The results show that TIMP-1-GPI blocked the secretion of MMP-7 by SW480 cells (Figure 1C). Thus, analogous to what was found for MMP-9 in the zymography assay, GPI-anchored TIMP-1 protein was found to reduce the secretion of MMP-7 into the growth media.

The reduced secretion of MMP-9 and MMP-7 suggested a potential sequestering of the proteins on the cell surface. FACS analysis using MMP-9 and MMP-7 specific monoclonal antibodies showed an increased signal for the proteins on the cell surface following treatment with 7 or $14 \mathrm{ng} / \mathrm{ml}$ TIMP-1-GPI (Figure 1D) but not with recombinant hTIMP-1 or denatured TIMP-1-GPI (14 ng/ $\mathrm{ml}$ ), with vehicle or with GPI-anchor/phosphatidylserine control (data not shown). The protein appeared to remain on the cell surface as the surface detection of TIMP-1GPI by FACS remained over a $72 \mathrm{~h}$ period (Figure 1E).

Additional control qPCR studies were performed (as described in Djafarzadeh et al., 2006) to determine if the suppressive effect of TIMP-1-GPI on MMP-7 or MMP-9 protein secretion could be explained in part by decreased gene expression. The results showed that mRNA levels were either largely unchanged (MMP-7) or were increased (MMP-9) with TIMP-1-GPI treatment at $24 \mathrm{~h}$ (data not shown).

We have previously shown that treatment with TIMP1-GPI can either enhance or suppress cell proliferation depending on the specific cell type studied (Djafarzadeh et al., 2004). To assess the effect of TIMP-1 surface engineering on the proliferation of SW480 cells, MTT assays were performed (Djafarzadeh et al., 2006). The exogenously added TIMP-1-GPI protein was found to elicit a dose-dependent decrease in the proliferation of SW480 (Figure 2A).

A reduction in the secretion of MMP-7 and MMP-9 suggests a reduced capacity of the SW480 cell line to undergo ECM invasion. The effect of TIMP-1-GPI vs. rhTIMP-1 treatment on the ability of SW480 cells to invade Matrigel was measured using induced migration to fetal calf serum (FCS) (Biocrom, Berlin, Germany), vascular endothelial growth factor (VEGF) (R\&D systems, Minneapolis, USA), and EGF (Biochrom, Berlin, Germany) using a modified Boyden chamber (Djafarzadeh et al., 2004, 2006). Cells which had undergone migration to the bottom of the insert were detached, lysed, and detected by CyQuant dye as described previously (Djafarzadeh et al., 2004, 2006). Optimal SW480 migration/ invasion was observed at $10 \% \mathrm{FCS}, 4 \mathrm{ng} / \mathrm{ml}$ VEGF, and $4 \mathrm{ng} / \mathrm{ml}$ EGF (data not shown). The effect of treatment with 2-14 ng/ml TIMP-1-GPI and with $14 \mathrm{ng} / \mathrm{ml}$ control rhTIMP-1 on SW480 invasion through Matrigel was then determined. The maximal migration to FCS, VEGF, and EGF were each set as $0 \%$ inhibition. 100\% inhibition of migration was set to the migration level obtained with unstimulated SW480 cells. The resultant effect of rhTIMP or TIMP-1-GPI treatment on migration was calculated as percent change in the 'maximal' value (Figure 2B). Treatment of the SW480 cells with increasing amounts of TIMP-1-GPI showed a dose-dependent suppression of invasion, while no effect was observed with rhTIMP-1 control protein.

The in vitro results suggested that in vivo treatment of a tumor environment with TIMP-1-GPI may directly influence tumor biology by reducing tumor proliferation and suppressing invasion into surrounding tissue. This was tested by the transplantation of SW480 tumors into immunodeficient mice.

SW480 cells were implanted under the skin of Rag (-/-) common $\gamma$ chain (-/-) C57BL/6 mice and the growth characteristics of the tumor established by monitoring growth by palpation. When the tumor size had reached approximately $0.5 \mathrm{~cm}$ in diameter, the animals were randomized into three groups and therapy was initiated. Peritumoral injection of $100 \mu \mathrm{l}$ of TIMP-1-GPI at $14 \mathrm{ng} /$ $\mathrm{ml}, 14 \mathrm{ng} / \mathrm{ml}$ of control rhTIMP-1 protein or vehicle ( $1 \times$ phosphate buffered saline, PBS, $0.025 \%$ Triton X$100 \mathrm{H}$ ) was applied. The injections were made every other day around the tumor (4 times $25 \mu$ l each) for a total of five treatments over a span of 10 days. One day following the last treatment, the animals were sacrificed and the tumor size measured. The results show that treatment 
A
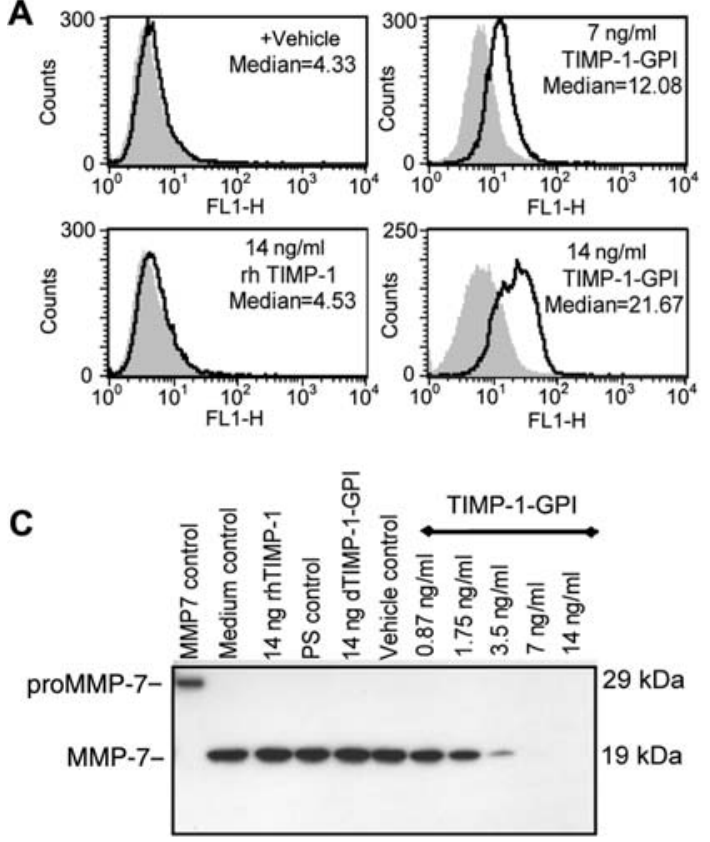

B

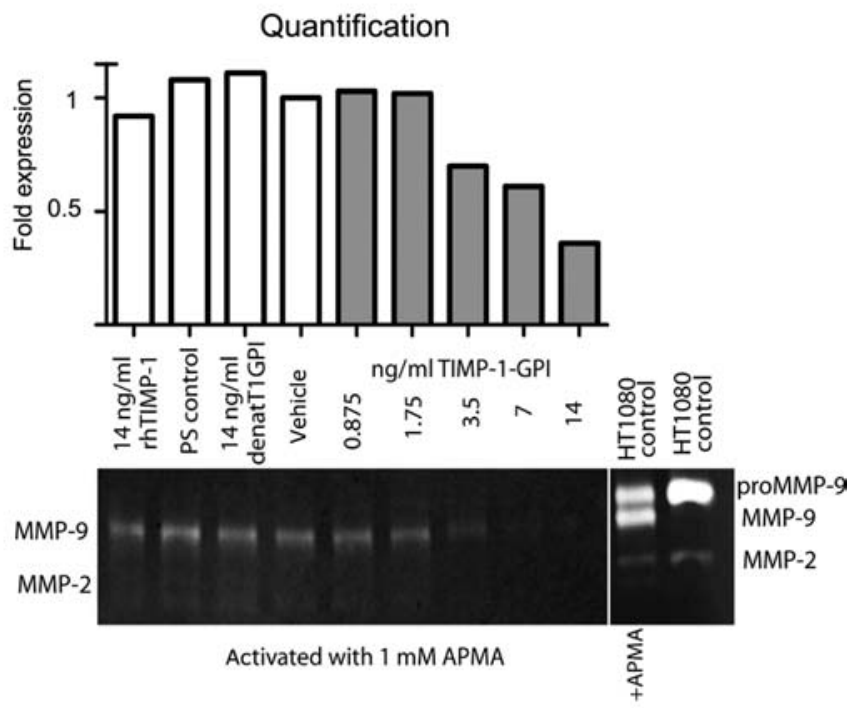

D
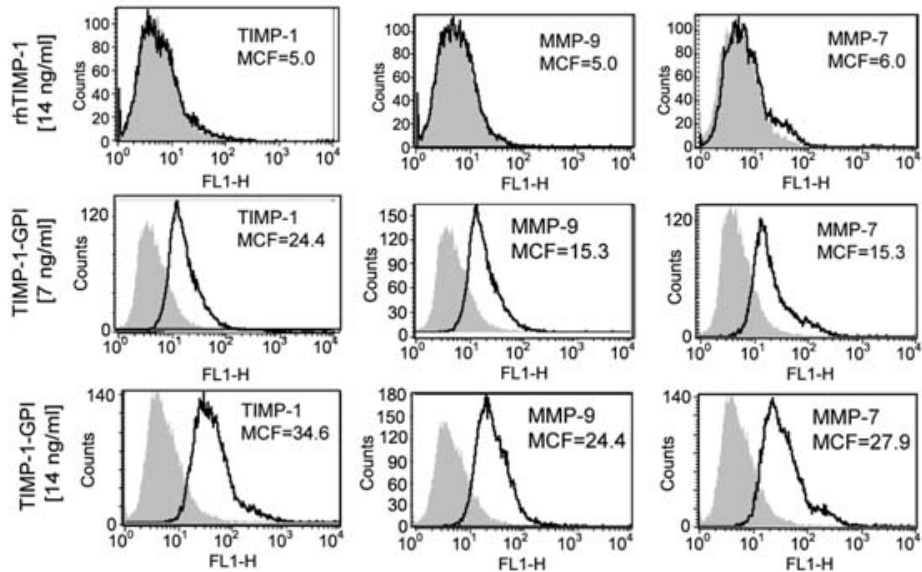

E
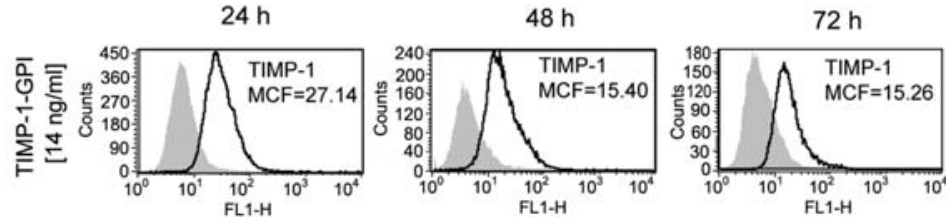

Figure 1 Incorporation of TIMP-1-GPI into SW480 cells and subsequent effect on MMP-9 and MMP-7 secretion.

The TIMP-1-GPI protein was produced, purified, and quantified as previously described (Djafarzadeh et al., 2004). The vehicle for all studies was $1 \times$ PBS with $0.025 \%$ Triton X-100H. SW480 cells (ATCC, Manassis, UT, USA; no. CCL-228) were cultured in Dulbecco's modified Eagle's medium with Glutamax-1 (Gibco-BRL/Life Technologies GmbH, Eggenstein, Germany) supplemented with 7.5\% FCS. To demonstrate reincorporation of TIMP-1-GPI protein into cell membranes, 14 ng/ml of TIMP-1-GPI, or control rhTIMP-1, was added to SW480 cells as described previously (Djafarzadeh et al., 2006). (A) TIMP-1 was detected on the cell surface by FACS analysis (Klier et al., 2001). Gray histograms represent isotype control stain; solid line histograms represent TIMP-1 antibody staining. (B) Gelatinase zymography (Djafarzadeh et al., 2004) showed that secretion of MMP-9 from SW480 was blocked by treatment with increasing levels of TIMP-1-GPI. Culture media (serum-free) were taken $48 \mathrm{~h}$ after treatment of SW480 with $14 \mathrm{ng} / \mathrm{ml}$ of rhTIMP-1, TIMP-1-GPI, phosphatidylserine, or denatured TIMP-1-GPI and compared to untreated control cells and medium and analyzed by gelatinase zymography (Djafarzadeh et al., 2004). Before analysis, culture supernatants were pretreated with $1 \mu \mathrm{M}$ APMA to induce activation of MMP-9 prior to loading on the gel. The band intensities were quantified using ImageJ (http://rsbweb.nih.gov/ij/). (C) Western blot using monoclonal antibody directed against human MMP-7 (Thermo Scientific, Fremont, CA, USA) was used to detect secretion from SW480 essentially as described previously (Djafarzadeh et al., 2006). Culture media (serum-free) were taken $24 \mathrm{~h}$ after treatment of SW480 with $14 \mathrm{ng} / \mathrm{ml}$ of rhTIMP-1, TIMP-1-GPI, phosphatidylserine, or denatured TIMP-1-GPI and compared to untreated control cells and medium. Lane loading was adjusted by monitoring absolute cell numbers and volume of growth media used. The detection antibody was a polyclonal rabbit anti-mouse-HRP (Dako, Glostrup, Denmark; diluted 1:5000). (D) FACS analysis shows a corresponding increase in surface expression of MMP-9 and MMP-7 following treatment with either $7 \mathrm{ng} / \mathrm{ml}$ or $14 \mathrm{ng} / \mathrm{ml}$ TIMP1-GPI. Control experiments using equal molar concentrations of GPI-anchor/phosphatidylserine, denatured TIMP-1-GPI (14 ng/ml), or vehicle did not lead to an increase in median channel fluorescence (data not shown). (E) The surface signal for TIMP-1-GPI (14 $\mathrm{ng} / \mathrm{ml}$ ) observed with FACS was stable for up to $72 \mathrm{~h}$. 
A

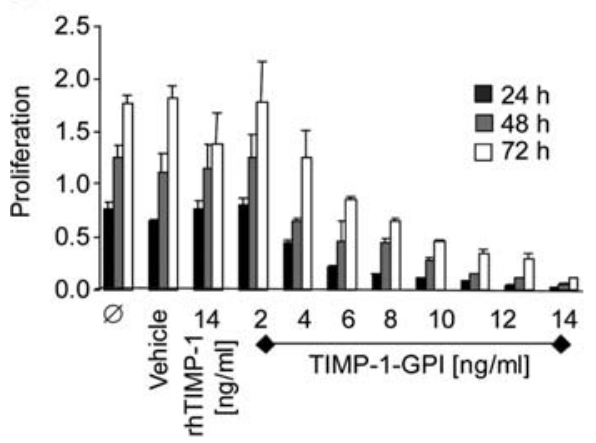

B

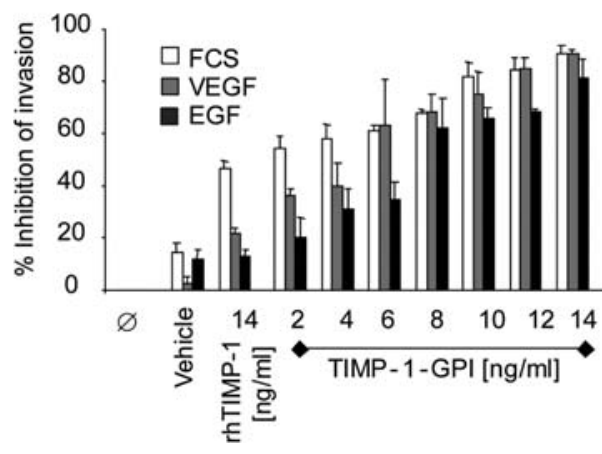

Figure 2 The GPI-anchored TIMP-1 suppresses proliferation of SW480 cells and inhibits tumor invasion.

The effect of cell surface engineering with TIMP-1-GPI on SW480 invasion through the Matrigel basement membrane model was assessed. Optimal invasion of the SW480 cells in response to $10 \% \mathrm{FCS}, 4 \mathrm{ng} / \mathrm{ml}$ EGF, or $4 \mathrm{ng} / \mathrm{ml}$ VEGF were set to 'zero', and the $100 \%$ inhibition value was set to the migration level observed with untreated SW480 cells (Djafarzadeh et al., 2004). $5 \times 10^{3}$ SW 480 cells per $100 \mu$ l medium were cultured in 96-well microtiter plates for $24 \mathrm{~h}$ under standard conditions to yield firmly attached and stably growing cells (Djafarzadeh et al., 2006). The SW480 cells were then pretreated with 2, 4, 6, 8, 10, 12 , and $14 \mathrm{ng} / \mathrm{ml}$ of TIMP-1-GPI or $14 \mathrm{ng} / \mathrm{ml}$ of rhTIMP-1 as described previously (Djafarzadeh et al., 2006). After $1 \mathrm{~h}$ the cells were washed. (A) The effect of increasing levels of TIMP-1-GPI or rhTIMP-1 control protein on the proliferation of SW480 was measured using an MTT assay (Djafarzadeh et al., 2006). MTT was added after $24 \mathrm{~h}, 48 \mathrm{~h}$, or $72 \mathrm{~h}$ as indicated. (B) The effect on the ability of SW480 to invade Matrigel was measured using a modified Boyden chamber as described earlier (Djafarzadeh et al., 2004, 2006).

with TIMP-1-GPI, but not with rhTIMP-1 or vehicle, lead to a profound reduction in tumor growth (Figure 3).

Cell surface engineering with TIMP-1-GPI has been shown to suppress renal cell carcinoma proliferation, migration, and invasion in vitro (Djafarzadeh et al., 2006). It is known that the tumor stroma can have a profound influence on the growth of tumors (Cox and O'Byrne, 2001; McCawley and Matrisian, 2001; Almholt and Johnsen, 2003). The effect of peritumoral application of TIMP1-GPI on the growth of colon carcinoma was assessed using a transplanted colon carcinoma tumor cell model implanted into Rag (-/-) common $\gamma$ chain (-/-) Balb/c mice. Application of TIMP-1-GPI in the absence of an adaptive immune response led to a significant reduction in tumor growth, while treatment with control rhTIMP-1 did not show a significant effect. These results provide further evidence that cell surface engineering with GPI-

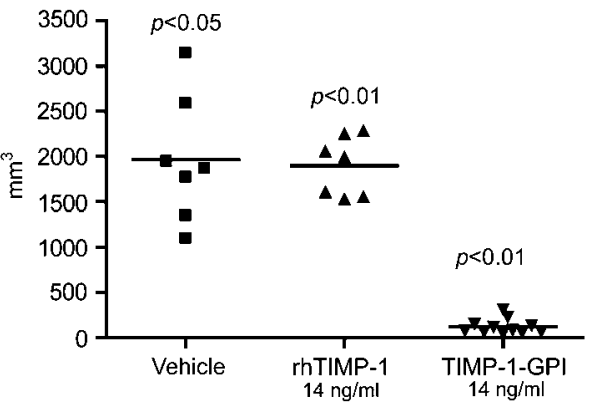

Figure 3 Effect of peritumoral application of TIMP-1-GPI on tumor growth.

Immunodeficient Rag (-/-) common $\gamma$ chain (-/-) C57BL/6 mice were inoculated with SW480 human colonic cancer cells at the upper dorsal aspect of the vertebral column under anesthesia. The effect of peritumoral and tumoral TIMP-1-GPI, rhTIMP-1, or vehicle treatment of the tumor was assessed. Tumor volume was detected using a caliper gauge (Helio-Point; Helios, Niedernhall, Germany). Measurement was performed in all three dimensions. The volume was then determined using the elongated ellipse method formula: $V=$ length $\times$ width $\times$ height $+p i / 6$. Statistical analysis was performed using the software SPSS version 16 (SPSS, Chicago, IL, USA). After normal distribution of the values was tested with the Kolmogorov-Smirnov test, the Student t-test was performed to test the differences between each of the three groups. Data are the mean of quadruplicate determinations and 95\% confidence interval. Each experiment was repeated at least three times.

anchored TIMP-1 leads to enhanced and unique activities, and that the GPI-anchored recombinant agent may represent a potential agent for therapeutic application, e.g., in the context of surgical resection to limit tumor regrowth.

\section{Acknowledgments}

This work was supported by grants from the Deutsche Forschungsgemeinschaft (NE468/2-4 and SFB-TR36 B6) to P.J.N. We thank Dr. Robert Lowe for his assistance with the graphical representations.

\section{References}

Almholt, K. and Johnsen, M. (2003). Stromal cell involvement in cancer. Recent Results Cancer Res. 162, 31-42.

Chirco, R., Liu, X.W., Jung, K.K., and Kim, H.R. (2006). Novel functions of TIMPs in cell signaling. Cancer Metastasis Rev. 25, 99-113.

Cockett, M.I., Birch, M.L., Murphy, G., Hart, I.R., and Docherty, A.J. (1994). Metalloproteinase domain structure, cellular invasion and metastasis. Biochem. Soc. Trans. 22, 55-57.

Cox, G. and O'Byrne, K.J. (2001). Matrix metalloproteinases and cancer. Anticancer Res. 21, 4207-4219.

Djafarzadeh, R., Mojaat, A., Vicente, A.B., von Luttichau, I., and Nelson, P.J. (2004). Exogenously added GPI-anchored tissue inhibitor of matrix metalloproteinase-1 (TIMP-1) displays enhanced and novel biological activities. Biol. Chem. 385, $655-663$

Djafarzadeh, R., Noessner, E., Engelmann, H., Schendel, D.J., Notohamiprodjo, M., von Luettichau, I., and Nelson, P.J. (2006). GPI-anchored TIMP-1 treatment renders renal cell carcinoma sensitive to FAS-mediated killing. Oncogene 25, 1496-1508. 
Djafarzadeh, R., Milani, V., Rieth, N., von Luettichau, I., Skrablin, P.S., Hofstetter, M., Noessner, E., and Nelson, P.J. (2009). TIMP-1-GPI in combination with hyperthermic treatment of melanoma increases sensitivity to FAS-mediated apoptosis. Cancer Immunol. Immunother. 58, 361-371.

Klier, C.M., Nelson, E.L., Cohen, C.D., Horuk, R., Schlondorff, D., and Nelson, P.J. (2001). Chemokine-induced secretion of gelatinase B in primary human monocytes. Biol. Chem. 382, 1405-1410.

Maskos, K. and Bode, W. (2003). Structural basis of matrix metalloproteinases and tissue inhibitors of metalloproteinases. Mol. Biotechnol. 25, 241-266.

Matsuyama, Y., Takao, S., and Aikou, T. (2002). Comparison of matrix metalloproteinase expression between primary tumors with or without liver metastasis in pancreatic and colorectal carcinomas. J. Surg. Oncol. 80, 105-110.

McCawley, L.J. and Matrisian, L.M. (2001). Tumor progression: defining the soil round the tumor seed. Curr. Biol. 11, R25R27.

Medof, M.E., Nagarajan, S., and Tykocinski, M.L. (1996). Cellsurface engineering with GPI-anchored proteins. FASEB J. 10, 574-586.

Mimori, K., Yamashita, K., Ohta, M., Yoshinaga, K., Ishikawa, K., Ishii, H., Utsunomiya, T., Barnard, G.F., Inoue, H., and Mori, M. (2004). Coexpression of matrix metalloproteinase-7 (MMP-7) and epidermal growth factor (EGF) receptor in colorectal cancer: an EGF receptor tyrosine kinase inhibitor is effective against MMP-7-expressing cancer cells. Clin. Cancer Res. 10, 8243-8249.

Murray, J.C., Symonds, P., Ward, W., Huggins, M., Tiga, A., Rice, K., Heng, Y.M., Todd, I., and Robins, R.A. (2004). Colorectal cancer cells induce lymphocyte apoptosis by an endothelial monocyte-activating polypeptide-II-dependent mechanism. J. Immunol. 172, 274-281.

Mylona, E., Kapranou, A., Mavrommatis, J., Markaki, S., Keramopoulos, A., and Nakopoulou, L. (2005). The multifunctional role of the immunohistochemical expression of MMP-7 in invasive breast cancer. Acta Pathol. Microbiol. Immunol. Scand. 113, 246-255.
Nagase, H., Visse, R., and Murphy, G. (2006). Structure and function of matrix metalloproteinases and TIMPs. Cardiovasc. Res. 69, 562-573.

Nagashima, Y., Hasegawa, S., Koshikawa, N., Taki, A., Ichikawa, Y., Kitamura, H., Misugi, K., Kihira, Y., Matuo, Y., Yasumitsu, H., et al. (1997). Expression of matrilysin in vascular endothelial cells adjacent to matrilysin-producing tumors. Int. J. Cancer 72, 441-445.

Oshima, T., Akaike, M., Yoshihara, K., Shiozawa, M., Yamamoto, N., Sato, T., Yamada, R., Fujii, S., Rino, Y., Kunisaki, C., et al. (2008). Clinicopathological significance of the gene expression of matrix metalloproteinase- 7 , insulin-like growth factor-1, insulin-like growth factor-2 and insulin-like growth factor-1 receptor in patients with colorectal cancer: insulinlike growth factor-1 receptor gene expression is a useful predictor of liver metastasis from colorectal cancer. Oncol. Rep. 20,359-364.

Rath, T., Roderfeld, M., Graf, J., Wagner, S., Vehr, A.K., Dietrich, C., Geier, A., and Roeb, E. (2006). Enhanced expression of MMP-7 and MMP-13 in inflammatory bowel disease: a precancerous potential? Inflamm. Bowel Dis. 12, 1025-1035.

Shinkai, Y. and Alt, F.W. (1994). CD3 epsilon-mediated signals rescue the development of $\mathrm{CD} 4^{+} \mathrm{CD}^{+}$thymocytes in RAG$2^{-/-}$mice in the absence of TCR beta chain expression. Int. Immunol. 6, 995-1001.

Strand, S., Vollmer, P., van den Abeelen, L., Gottfried, D., Alla, V., Heid, H., Kuball, J., Theobald, M., Galle, P.R., and Strand, D. (2004). Cleavage of CD95 by matrix metalloproteinase-7 induces apoptosis resistance in tumour cells. Oncogene 23, 3732-3736.

Visse, R. and Nagase, H. (2003). Matrix metalloproteinases and tissue inhibitors of metalloproteinases: structure, function, and biochemistry. Circ. Res. 92, 827-839.

Yoshimoto, M., Itoh, F., Yamamoto, H., Hinoda, Y., Imai, K., and Yachi, A. (1993). Expression of MMP-7 (PUMP-1) mRNA in human colorectal cancers. Int. J. Cancer 54, 614-618.

Received December 11, 2008; accepted May 7, 2009 